\title{
Über die Atembewegungen des Kaninchens während der Erstickung.
}

\author{
Von \\ Tamon Hoshi. \\ (星 多 聞) \\ (Aus dem Pharmakologischen Institut der Tohoku Universität \\ zu Sendai. Direktor: Prof. S. Yagi.)
}

Dass sowohl die Abnahme des Sauerstoffs als auch die Anhäufung der Kohlensäure im Blute auf das Respirationszentrum erregend wirken, ist seit den Untersuchungen von Pflüger, ${ }^{1)} \mathrm{Zuntz}$ und Loew $\left.y,{ }^{2}\right) \mathrm{Hald}$ ane und Priestle ${ }^{3)}$ und mehreren anderen eine bekannte Tatsache. Ich wollte mich zuerst mit der Frage beschäftigen, in welcher Reihenfolge die durch den Sauerstoffmangel und die Kohlensäureanhäufung im Blute verursachten Atemveränderungen ablaufen, ohne dabei aber auf die Frage einzugehen, auf welcher der beiden Bedingungen die Veränderungen beruhen. Während mehrere Mitteilungen über die Wirkung von Kohlensäure auf die Atmung vorhanden sind, liegen solche uiber die Erstickung bisher weniger zahlreich vor; unter ihnen ist besonders die von $\mathrm{Hög} \mathrm{yes}^{4}$ als die eingehendste zu nennen. In seinen Untersuchungen wurde in die Luftröhre eines Kaninchens eine Glaskanüle mit zwei Ästen eingeführt, von denen der eine mit einem Tambour verbunden wurde, der andere mit der Luft kommunizierte und durch einen angebrachten Hahn verschlossen werden konnte. Er fand, dass die Atembewegungen nach dem Verschluss der Luftröhre in verschiedenen Fällen regelmässig dieselben Veränderungen erlitten. Sie bestanden darin, dass zuerst eine Vertiefung der Atemzüge eintrat, welche hauptsächlich die Inspiration betraf, worauf sich dann eine exspiratorische Anstrengung hinzugesellte, welche sich bis zur krampfhaften steigerte; nach vorübergehender vollständiger Atemruhe in Erschlaffung erfolgten wieder

\footnotetext{
1) Pflüger, Arch. f. d. ges. Physiol., 1867, 1, 61.

2) Zuntz und Loewy, Arch. f. (Anat. u.) Physiol., 1897, 379.

3) Haldane und Priestley, Journ. of Physiol., 1905, 32, 225.

4) Högyes, Arch. f. exp. Path. u. Pharm., 1876, 5, 86.
} 
einige tiefe Atembewegungen mit aktiven In- und passiven Exspirationen, um bald dem Atemstillstand Platz zu machen. In anderen Untersuchungen fand er auch, dass eine vorherige Dur'chschneidung der $\mathrm{N}$. vagi nur eine geringe Abweichung von dem erwähnten Verlauf verursachte, während Exstirpation des Vierhügels samt dem Sehhügel und Grosshirn nur eine inspiratorische Dyspnoe bewirkte, welche bis zum Stillstand immer seltner und nur einmal von einer etwa 30 Sekunden langen Atempause unterbrochen wurde, aber keine exspiratorische Anstrengung verursachte. Trotz seiner genauen Untersuchungen schien es nötig diese noch zu ergänzen, da bei allen seinen Versuchen der Verlauf der Erstickung sehr kurz, nicht länger als 4 Minuten dauerte, sodass die einzelnen Atemveränderungen vielleicht nicht deutlich genug in Erscheinung getreten sein könnten. Dies gab mir Anlass einige Untersuchungen darüber anzustellen.

Da die Wirkung der Kohlensäure auf die Atmung, nach Zuntz und Loewy," nur dann deutlich in Erscheinung tritt, wenn die Atmung unbehindert vor sich gehen kann, so wurde in meinen Versuchen auch darauf geachtet, dass mechanische Hindernisse im Luftwege, etwa solche wie bei den Versuchen von Högyes, möglichst vermieden wurden. So wurde die Schnauze eines Kaninchens, welches in den früher bereits beschriebenen Atemkasten ${ }^{2)}$ eingebracht worden war, in eine passende Kappe gesteckt, welche an ihrer Spitze mit zwei kurzen, $2 \mathrm{~cm}$ langen Glasröhren ausgerüstet war, von denen die eine mit einem gleich weiten, $2 \mathrm{~m}$ langen Glasrohr verbunden und die andere eventuell mit einem Stöpsel verschlossen werden konnte. Bei Offenbleiben des letzteren Glasrohrs konnte das Tier ohne besondere Schwierigkeiten die Atmung stundenlang fortsetzen, aber bei Verschliessen desselben entwickelten sich allmählich die Erstickungserscheinungen, trotzdem die Atembewegungen wegen Offenbleibens des anderen Glasrohrs ohne Hindernis vor sich gehen sollten. Bei wiederholten Versuchen wurde gefunden, dass die Zeitdauer, während welcher die Erstickungserscheinungen abliefen, bei den einzelnen Versuchstieren ziemlich verschieden war, indem sie von 40 bis 80 Minuten betrug. Dessenungeachtet war in die Reihenfolge der Erstikkungserscheinungen kein wesentlicher Unterschied zwischen den einzelnen Versuchstieren bemerkbar.

Kurz nach dem Verschluss des kurzen Luftweges begann die Atmung an Tiefe zuzunehmen; diese Vertiefung, welche nicht nur durch die Verstärkung der Inspiration, sondern auch durch die der Exspiration bedingt war, wurde immer ausgeprägter. Die Frequenz erlitt

1) Zuntz und Loewy, l.c.

2) Hos hi, Tohoku Journ. Exp. Med., 1932, 18, 559. 


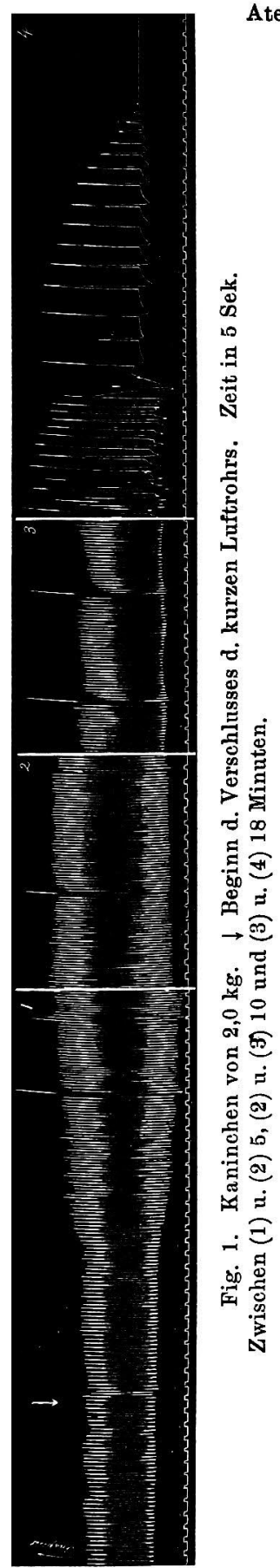

zuerst keine Veränderung, indem sie erst 2 bis 5 Minuten nach dem Verschluss zuzunehmen begann. Mit der Zeit wurde die Atmung jedoch immer frequenter. So erreichten die Tiefe und Frequenz gegen den Zeitabschnitt, während welchem Zyanose der Ohren und Veränderung der Pupillen am deutlichsten zum Vorschein kamen, den höchsten Grad, indem die erstere 50 bis 200 Prozent und die letztere 50 bis 100 Prozent des ursprünglichen normalen Wertes zunahmen. Danach nahmen die beiden ganz allmählich ab, ohne aber zu dem normalen Wert zurückzukehren, bis zu jener Zeit, wo die Pupillen sich am stärksten erweiterten. Dann fing die Frequenz an ziemlich rasch abzunehmen, um nach einigen Minuten zum vollständigen Atemstillstand zu führen, während die Tiefe sowohl in inspiratorischer als auch in exspiratorischer Exkursion noch einmal etwas zunahm, bevor sie, erst in exspiratorischer und dann auch in inspiratorischer Hinsicht, bis zum vollständigen Verschwinden abnahm. Bei der letztgenannten Zunahme der Atemexkursion war bemerkbar, dass jede Inspiration nicht gleich aus der vorausgehenden Exspiration hervorging, sondern erst nach kürzerer oder längerer Atemverhaltung in aktiver Exspirationsstellung erfolgte, welche immer einem allgemeinen Krampfanfall entsprach. Zur besseren Veranschaulichung füge ich Fig. 1 hinzu. Das Atemvolumen pro Minute vermehrte sich zuerst infolge der Zunahme des einzelnen Atemvolumens und später ausserdem noch infolge der der Atemfrequenz, sodass es in einem gewissen Stadium das Doppelte der Norm oder darüber erreichen konnte; es verminderte sich dann, anfänglich allmählich, aber später ziemlich schnell bis zum vollständigen Verschwinden; dieser Verlauf wurde noch einmal von einer leichten Zunahme unterbrochen. Um die gegenseitige Beziehung dieser einzelnen Atemveränderungen während der Erstickung 


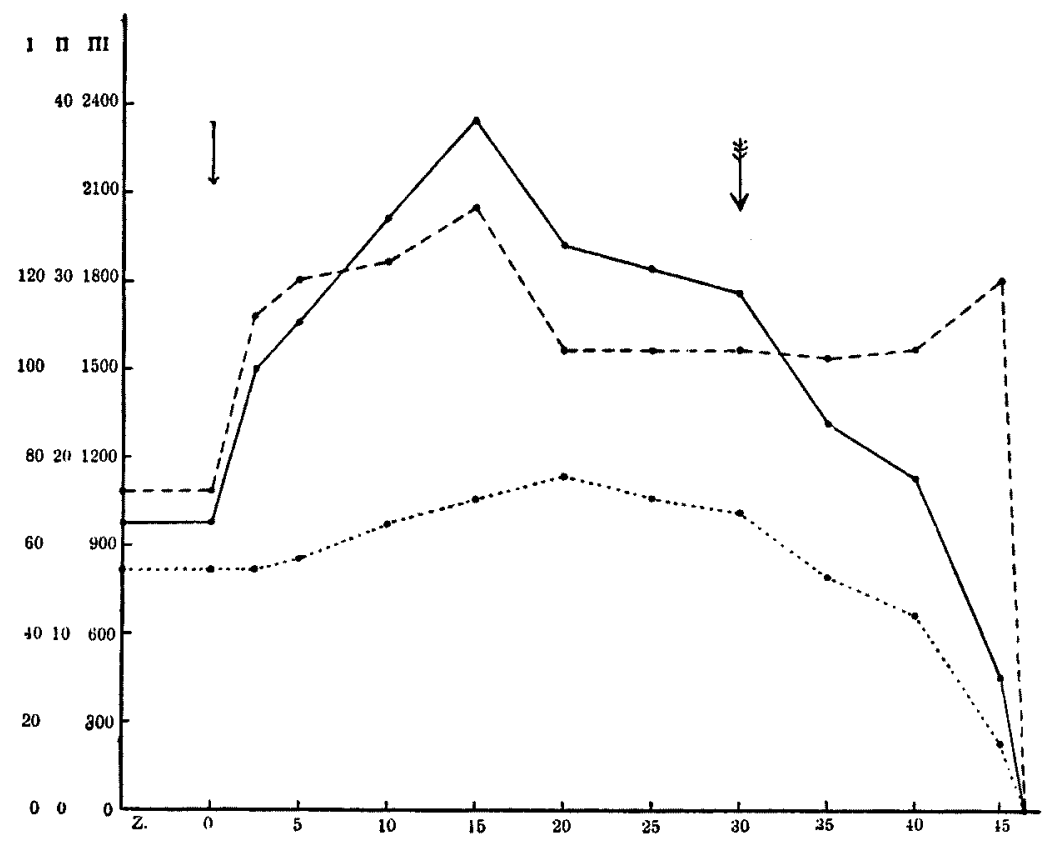

Fig. 2. Kaninchen von $2,0 \mathrm{~kg}$.

I ...... Atemzahl pro Minute.

II -.. Durchschnittsvolum d. einzel. A temzüge in ccm.

III - Atemvolum pro Minute in $\mathrm{ccm}$.

Z. Zeit n. d. Verschluss d. kurzen Luftrohrs.

$\downarrow$ Beginn d. Verschlusses.

Auftreten d. ersten Krampfanfalls.

zu zeigen, sei hier Fig. 2 angeführt.

Diese Ergebnisse bestätigen somit die von $H$ ögyes, jedoch nicht in allen Punkten, indem sie zeigen, dass bei der Erstickung Vertiefung der Atemzüge in den Vordergrund tritt, welche nicht bloss die Inspiration, sondern auch die Exspiration betrifft, im späteren Stadium in Verflachung mit aktiver In- und passiver Exspiration übergeht, um schliesslich zu verschwinden, und dass dabei auch eine Beschleunigung der Atmung eintritt, aber weit später als der Beginn der Vertiefung; und etwas früher verschwindet als der Beginn der Verflachung.

Bei Kaninchen, deren Hirnstamm vor etwa 5 Stunden dicht hinter dem Vierhügel und deren Vagi gleichzeitig durchschnitten worden waren, führte der Verschluss des kurzen Luftwegs zu einem etwas anderem Bild als bei den unversehrten Tieren, indem die Atmung von Anfang an an Frequenz einbüsste, sodass die Atemzüge bis zu ihrem vollständigen Verschwinden immer seltner wurden, ohne dass diese 
Reihenfolge einmal von der Beschleunigung unterbrochen wurde, die bei den unversehrten niemals fehlte, wie aus Fig. 3 ersichtlich ist.
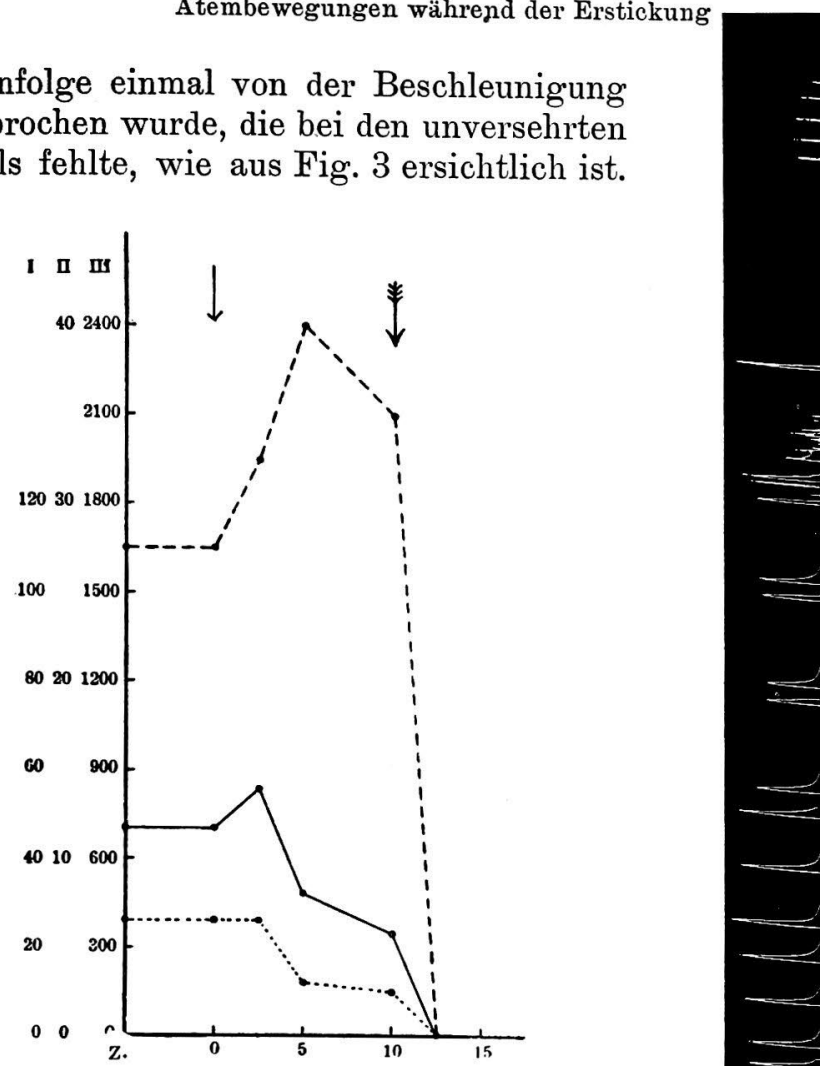

Fig. 3. Kaninchen von 1,9 kg. Vor 5 Stunden beide Grosshirnhemisphären entfernt und Vagi durchschnitten. I ...... Atemzahl pro Minute.

II - - Durchschnittsvolum d. einzel. A temzüge in ccm. III- Atemvolum pro Minute in ccm.

Z. Zeit n. d. Verschluss d. kurzen Luftrohrs.

$\downarrow$ Beginn d. Verschlusses.

Auftreten d. ersten Krampfanfalls.

An Tiefe nahm die Atmung auch hierbei infolge der Verstärkung der Inspiration sowie, im Gegensatz mit der Angabe von Högyes, der Verstärkung der Exspiration immer mehr zu und fing dann wieder abzunehmen an, um zuletzt dem Atemstillstand überzugehen, wie Fig. 4 zeigt. Die Zeitdauer, in welcher die Erstickungserscheinungen abliefen, war im allgemeinen beträchtlich kürzer als diejenige bei den unversehrten Tieren, indem sie durchschnittlich etwa 10 Minuten betrug. 
Aus obigen Versuchen geht bündig hervor, einerseits dass das Erstickungsblut unmittelbar auf das Atemzentrum in der Medulla oblongata solche Wirkungen ausübt, dass die Atemfrequenz ab- und gleichzeitig die Atemtiefe zunimmt, und anderseits dass es mittelbar dasselbe Zentrum veranlasst durch seine Wirkung auf andere Gebiete als die Medulla oblongata, die Atembeschleunigung hervorzurufen. Dass die Erstickung der operierten Tiere einen kürzeren Verlauf als die der nicht operierten nimmt, kann vielleicht dadurch erklärt werden, dass bei den ersteren wegen Fehlen der Atembeschleunigung der Gasaustausch zwischen Luft und Blut schlechter als bei den letzteren stattfindet, sodass die Sauerstoffabnahme und Kohlensäurezunahme im Blute rascher einen solchen Grad erreicht, welcher die Erschöpfung des Atemzentrums zu verursachen vermag. 